\title{
Do non-steroidal anti-inflammatory drugs increase colonic permeability?
}

\author{
A P Jenkins, D R Trew, B J Crump, W S Nukajam, J A Foley, I S Menzies, B Creamer
}

\begin{abstract}
Urinary excretion of orally administered lactulose and 51 chromium labelled ethylenediamine tetra-acetate (51Cr-EDTA) was measured in 12 healthy adult subjects and in six patients with ileostomies to assess intestinal permeability. In normal subjects, 24 hour urinary recovery of 51Cr-EDTA was significantly greater than that of lactulose (mean (SEM) $2.27(0.15) v 0.50(0.08) \%$ oral dose; $\mathbf{p}<0.001)$, but in ileostomy patients recovery of the two markers was the same. In normal subjects, therefore, the difference between the two markers may arise from bacterial breakdown of lactulose but not of 51Cr-EDTA in the distal bowel, urinary excretion of lactulose representing small intestinal permeation and that of 51Cr-EDTA representing both small and large intestinal permeation. The markers were then given simultaneously to nine patients receiving non-steroidal antiinflammatory drugs (NSAIDs) for rheumatoid arthritis and osteoarthritis. The 24 hour urinary recovery of 51Cr-EDTA in the patients was significantly greater than normal (4.64 $(1.20) v 2.27(0.15) \%$ oral dose; $\mathbf{p}<0.01)$, but that of lactulose was not significantly affected. Moreover, the increase in 51Cr-EDTA recovery was most noticeable in the later urine collections. Both of these findings suggest that NSAIDs may increase colonic permeability.
\end{abstract}

Intestinal permeability refers to those properties of the mucosa that allow passive unmediated diffusion of molecules,' and permeation is the word used for the observed transfer across the mucosa. 51 Chromium-labelled ethylenediamine tetra-acetate (51Cr-EDTA) and lactulose both cross the mucosa entirely by passive nonmediated diffusion, perhaps via low incidence paracellular channels. ${ }^{2}$ As neither molecule is metabolised after absorption and both are fully excreted in the urine ${ }^{2}$ their urinary recovery after oral administration provides a convenient estimate of intestinal permeation. Increased permeation of both lactulose and 51Cr-EDTA occurs with small intestinal mucosal lesions such as Crohn's disease ${ }^{3+}$ and coeliac disease ${ }^{56}$ and also after ingestion of very hyperosmolar solutions and cetrimide. ${ }^{2}$

In patients receiving non-steroidal antiinflammatory drugs (NSAIDs) for rheumatoid or osteoarthritis there is increased intestinal permeation of $51 \mathrm{Cr}$-EDTA. ${ }^{7}$ The permeability change is caused by the NSAID treatment rather than the underlying disease, as it is not present in untreated patients. The site of the permeability change in the intestine, however, is uncertain. In patients receiving NSAIDs for more than six months there are abnormalities of indium labelled leucocyte scans that suggest diffuse small intestinal inflammation ${ }^{8}$; vitamin $B_{12}$ and bile acid absorption may also be impaired, and a few patients have terminal ileal strictures and ulceration seen on small bowel barium studies. ${ }^{8}$ But there is also evidence that NSAIDs may affect the colon as they may exacerbate colitis ${ }^{10}$ and cause rectal bleeding and colonic perforation in previously healthy subjects."

The effect of NSAIDs on the permeation of lactulose has not previously been established, but could be relevant to the site of the permeability change. Although 51Cr-EDTA and lactulose have similar molecular weights (340 and 342 respectively) and solubility characteristics, ${ }^{12}$ the 24 hour urinary recovery of $51 \mathrm{Cr}$ EDTA in normal subjects is greater than that of lactulose,,$^{213}$ and the difference becomes more noticeable towards the end of the collection period. ${ }^{2}$ This could be because lactulose, unlike 51Cr-EDTA, is degraded by bacterial flora of the distal gut. ${ }^{14}$ In support of this, the urinary recoveries of ingested $51 \mathrm{Cr}$-EDTA and lactulose were found to be closely similar in three patients with ileostomies. ${ }^{13}$ If these results can be confirmed in a larger group of patients, they suggest that urinary excretion of lactulose reflects small intestinal permeation, and that of 51Cr-EDTA both small intestinal and colonic permeation. Therefore simultaneous administration of both markers should distinguish changes involving the distal gut alone from those involving the more proximal intestine, where the bacterial activity is low.

The sequential measurement of markers excreted in urine over the 24 hours after ingestion should also help to distinguish proximal from distal intestinal permeability changes. Small intestinal lesions increase the recovery of the markers mainly in the early collection periods, ${ }^{24}$ while colonic lesions affect mainly the later recoveries. ${ }^{4}$

This study is in two parts. The first part attempts to confirm preliminary findings ${ }^{13}$ by comparing the urinary excretion of lactulose and 51Cr-EDTA in normal healthy subjects and in patients with ileostomies. The second part of the study examines the excretion of both markers and their time course of urinary recovery in patients receiving NSAIDs.

\section{Methods}

\section{EXPERIMENTAL PROTOCOL}

Lactulose $(5 \mathrm{~g})$ and $51 \mathrm{Cr}$-EDTA $(50 \mu \mathrm{Ci})$ were administered to all subjects in an iso-osmolar solution after an overnight fast. Simultaneously, each subject ingested two gelatin capsules each
The Rayne Institute, SEl 7EH.

Accepted for publication 26 March 1990 
containing $40 \mathrm{mg}$ of a non-absorbable blue dye used for food colouring (Brilliant Blue FCF, Pronk, Davis and Rusby Ltd, London N7). The time at which the blue dye first appeared in the faeces was used to compare total intestinal transit times for the normal control subjects and patients receiving NSAIDs.

All urine produced over the 24 hours after ingestion of the test solution was collected for sequential time periods $0-5,5-10$, and 10-24 hours in one litre plastic sample bottles, each containing 10 drops merthiolate $(10 \% \mathrm{w} / \mathrm{v})$ as preservative. Volumes were recorded and aliquots kept for analysis. Urinary lactulose was subsequently estimated by quantitative thin layer chromatography. This technique was originally developed to measure monosaccharides, ${ }^{15}$ but was subsequently modified to measure disaccharides ${ }^{16}$ : the minimum level of detection for most sugars is below $0.1 \mathrm{mmol} / \mathrm{l}$ and the assay gives a coefficient of variation, without replication, of between 2 and $8 \%$ over the test range of sugar concentrations. Urinary 51Cr-EDTA was estimated by counting $0.5 \mathrm{ml}$ aliquots of urine for radioactivity together with $0.5 \mathrm{ml}$ of a 1 in 200 dilution of the appropriate oral test solution. Excretion of lactulose and 51Cr-EDTA was expressed as a percentage of the orally administered dose.

The study was approved by the Ethical Committee of St Thomas's Hospital and all subjects gave informed consent.

\section{EXPERIMENT 1}

Twelve healthy adult volunteers (aged 22-58, median 28 years) and six patients with ileostomies (aged 33-66, median 51 years) were recruited. Three of the ileostomy patients had Crohn's disease and three had undergone total colectomy for ulcerative colitis. The three patients with Crohn's disease had had ileostomies formed for colonic disease, not more than five inches of terminal ileum being resected, and at the time of the experiment there was no evidence of active disease in the small intestine. From the ileostomy patients, in addition to the urine collection, all small bowel output was collected in consecutive time periods until the effluent no longer contained the ingested blue dye. The 51Cr-EDTA content of the effluent was measured.

\section{EXPERIMENT 2}

Nine patients (aged 45-72, median 48 years) receiving NSAIDs for rheumatoid arthritis (six) or osteoarthritis (three) were tested (see Table for type of NSAID and dosage). The duration of

Non-steroidal anti-inflammatory drug treatment in nine patients with rheumatoid or osteoarthritis

\begin{tabular}{llr}
\hline Non-steroidal anti-inflammatory drug & No patients & Daily dose \\
\hline Diclofenac & 3 & $50 \mathrm{mg} \times 2$ \\
Naproxen & 2 & $500 \mathrm{mg} \times 2$ \\
Flurbiprofen & 2 & $250 \mathrm{mg} \times 2$ \\
Indomethacin & $100 \mathrm{mg} \times 2$ \\
Piroxicam & 1 & $50 \mathrm{mg} \times 2$ \\
& 1 & $25 \mathrm{mg} \times 3$ \\
\end{tabular}

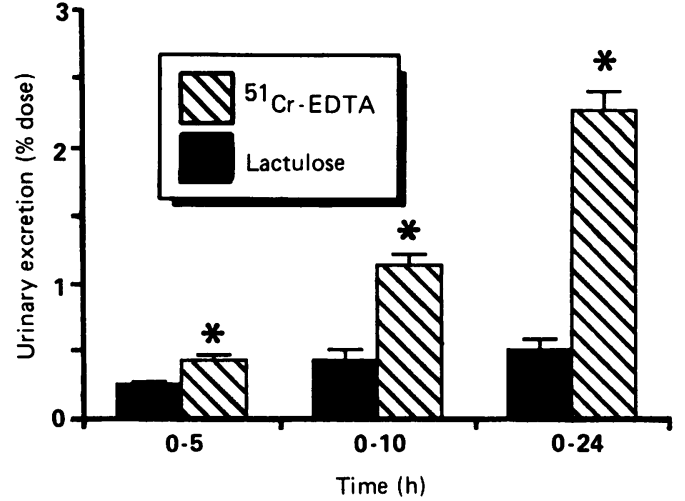

Figure 1: Mean (SEM) cumulative urinary recovery of lactulose and 51 chromium labelled ethylene diamine tetraacetate (51Cr-EDTA) in 12 normal subjects.

$\star 51 C r-E D T A$ differs from lactulose $(p<0.001)$.

treatment before the test was 13 days to 9 years, median 4 years. None of the patients were receiving slow release or rectal formulations of the drugs or sulphasalazine. Two of the patients with rheumatoid arthritis were also receiving penicillamine, one oral prednisolone, and one oral gold therapy. No patient had a history of intestinal disease.

\section{STATISTICAL METHODS}

The urinary recoveries of 51Cr-EDTA and lactulose in Experiment 1 were compared using Student's paired $t$ test. The urinary recoveries of lactulose and 51Cr-EDTA derived from Experiment 2 were compared with the values for normal subjects of Experiment 1 using Wilcoxon's test for unpaired samples. Results are expressed as means (SEM), unless otherwise indicated.

\section{Results}

\section{EXPERIMENT 1}

In the 12 normal subjects, the 24 hour urinary recovery of $51 \mathrm{Cr}$-EDTA was significantly greater than that of lactulose $(2 \cdot 27(0 \cdot 15) v 0.50$ $(0.08) \%$ oral dose, $\mathrm{p}<0.001)$. Figure 1 shows their cumulative urinary excretions during the course of 24 hours. A difference between the recovery of the two markers developed after five hours and became progressively greater after 10 and 24 hours. In 11 subjects, the blue dye transit marker did not appear in the faeces within 24 hours of ingestion of the test solution. In one

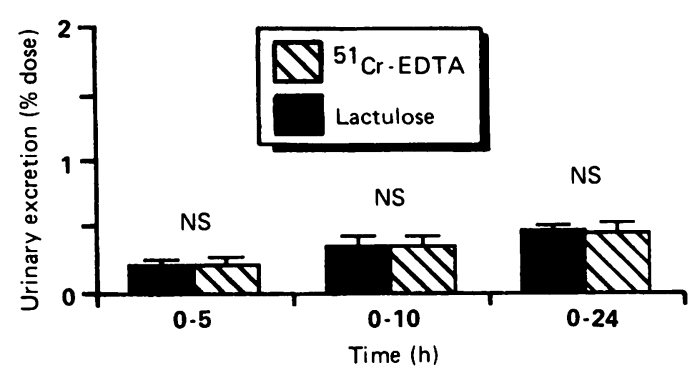

Figure 2: Mean (SEM) cumulative urinary recovery of lactulose and 51 chromium labelled ethylene diamine tetraacetate (51Cr-EDTA) in six patients with ileostomies.

$N S=$ no difference between $51 C r-E D T A$ and lactulose. 


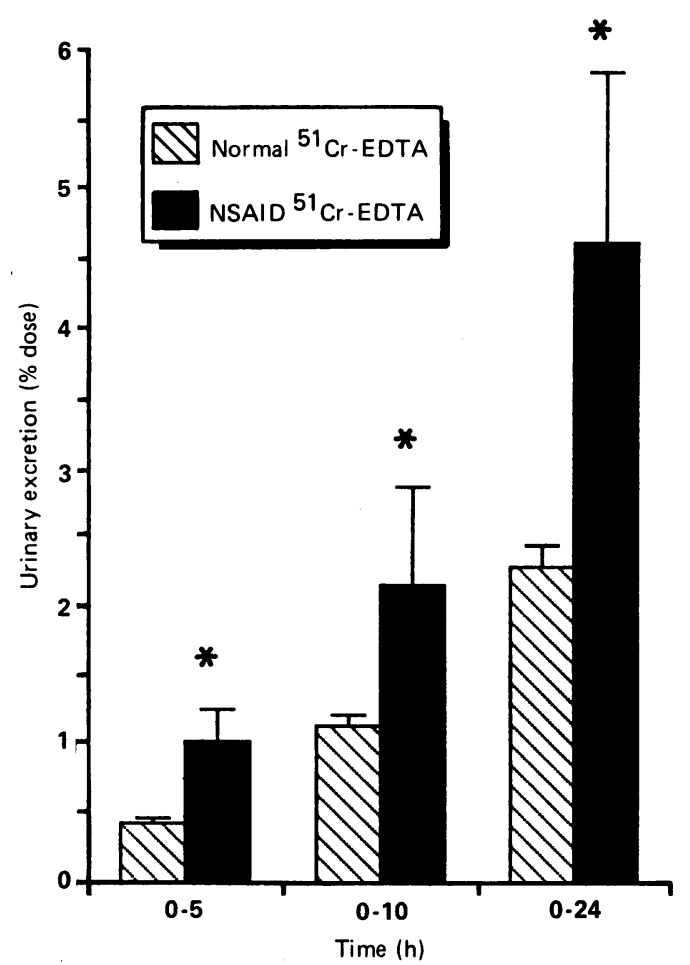

Figure 3: Mean (SEM) cumulative recovery of 51 chromium labelled ethylenediamine tetra-acetate (51Cr-EDTA) in 12 normal subjects and in nine patients receiving non-steroida anti-inflammatory drugs (NSAIDs).

${ }^{\star} N S A I D$ s differ from normal subjects $(p<0.01)$.

subject, blue dye was noted in the faeces eight hours after ingestion of the test solution and this subject had the lowest 24 hour urinary excretion of 51Cr-EDTA (1.54\%).

In the six patients with ileostomies, there was no significant difference between the 24 hour urinary recoveries of 51Cr-EDTA and lactulose (respectively $0.45(0.08)$ and $0.46(0.06) \%$ oral dose). The cumulative recoveries of the two markers (see Fig 2) showed no significant difference during any time period.

The recorded five hour ileostomy output of 51Cr-EDTA (mean (SD)) was 75.05 (16.21)\% oral dose, although, as collection of effluent was incomplete in some subjects, this probably underestimates the true five hour output.

\section{EXPERIMENT 2}

In the nine patients receiving NSAIDs, the mean 24 hour urinary recovery of 51Cr-EDTA was significantly increased compared with normal subjects $(4.64(1.20) v 2 \cdot 27(0 \cdot 15) \%$ oral dose, NSAIDs $v$ normal subjects, $\mathrm{p}<0 \cdot 01)$. There was, however, no significant increase in recovery of lactulose $(0.58(0.07) v 0.50(0.08) \%$ oral dose). The cumulative recovery of $51 \mathrm{Cr}$-EDTA is shown in Figure 3. At five hours, recovery of 51Cr-EDTA was increased above the normal value, but the increase was greater in the later collection periods.

In none of the subjects receiving NSAIDs did the blue dye transit marker appear in the faeces within 24 hours of ingesting the test solution.

\section{Discussion}

The possibility that simultaneous administration of lactulose and 51Cr-EDTA provides a means of assessing the site of intestinal permeability change has been investigated and the permeation of the two markers has been measured in patients receiving NSAIDs. Although our normal subjects were younger than members of the other two groups, this is unlikely to account for the observed changes. ${ }^{17}$

Our results confirm preliminary findings ${ }^{13}$ that the difference between the urinary recoveries of ingested 51Cr-EDTA and lactulose in normal subjects is not found in patients with ileostomies. Therefore, the increased uptake of 51Cr-EDTA compared with lactulose from the normal intestine may be largely of colonic origin, the most likely explanation being degradation of lactulose, but not of 51Cr-EDTA, by bacteria in the lower gut. The difference in urinary excretion, however, may not strictly correspond with colonic permeation because the terminal ileum has an active population of faecal type flora, ${ }^{18}$ and also because some lactulose could temporarily survive degradation after entering the colon. Furthermore, the intestine may sometimes be colonised mainly by non-lactulose fermenting organisms.

The increased permeation of 51Cr-EDTA in the patients receiving NSAIDs is consistent with previous findings. ${ }^{7}$ Four of our nine patients were also taking other drugs; it is uncertain whether these could have influenced the findings, but the 24 hour urinary excretion of $51 \mathrm{Cr}$ EDTA for the five subjects taking NSAIDs alone showed the same trend. An interesting new observation was that lactulose permeation was not increased by NSAID treatment. By contrast, permeation of both lactulose and 51Cr-EDTA is increased when small intestinal integrity is affected by coeliac disease ${ }^{19}$ or by ingested cetrimide. ${ }^{20}$ The increased permeation of $51 \mathrm{Cr}$ EDTA, but not lactulose, induced by NSAIDs suggests that these drugs increase permeability mainly in the lower intestine.

The time course of urinary 51Cr-EDTA excretion should reflect the site of permeability change, ${ }^{4}$ and our finding that NSAIDs increased mainly the late excretion of 51Cr-EDTA is consistent with enhanced permeation from the lower gut. Including lactulose in the oral test solution might affect the relative contributions of the small intestine and colon to permeation of 51Cr-EDTA by accelerating small intestinal transit. ${ }^{21}$ Our suggestion that enhanced late excretion of 51Cr-EDTA represents colonic permeation is still valid, however, since another study $^{2}$ showed that even when $5 \mathrm{~g}$ lactulose was included in the test solution a small intestinal lesion enhanced the early rather than the late permeation of 51Cr-EDTA. Although there was some increase in recovery of $51 \mathrm{Cr}$-EDTA during the initial five hour urine collection in our patients receiving NSAIDs, this does not necessarily represent enhanced small intestinal permeation. The data from our ileostomy patients suggest that a large proportion of the oral dose of 51Cr-EDTA may enter the colon within the initial five hour period.

A reasonable explanation for our findings is that NSAID therapy may most affect permeability in the lower intestine, where lactulose is 
rapidly degraded by bacteria before reaching the mucosal surface. It is therefore interesting that germ free rats show increased resistance to intestinal damage caused by large doses of NSAIDs. ${ }^{22}$ Many available NSAIDs undergo an enterohepatic circulation which involves intestinal bacterial breakdown of the inactive conjugate excreted in the bile ${ }^{23}$; it is possible that the active drug released from the conjugate could cause mucosal damage. In theory, NSAIDs could also enhance permeation of 51Cr-EDTA by prolonging colonic transit time relative to control subjects. This cannot, however, account for our results since only one of the control subjects excreted the blue dye transit marker in the stool within the 24 hour study period, and excluding this subject does not qualitatively alter the findings.

NSAIDs have been reported to exacerbate ulcerative colitis $^{910}$ and occasionally to cause rectal bleeding. " Our findings also suggest that the colon could be an important site for adverse reactions to these drugs.

We thank Dr D A H Yates for permission to study his patients, Mrs C Myers for help with recruiting ileostomy patients, and the
Special Trustees of St Thomas's Hospital for supporting this Special
work.

Part of this work was presented at the meetings of the Medical Research Society in July 1988 and January 1989. These presentations are published in abstract: Clin Sci 1988; 75 Suppl 19: 9 and Clin Sci 1989; 76 Suppl 20: 27.

1 Menzies IS. Transmucosal passage of inert molecules in health and disease. In: Skadhauge E, Heintze K, eds. Intestinol absorption and secretion. Lancaster: MTP Press, 1984: $527-$ 43 (Falk symposium 36 ).

2 Maxton DG, Bjarnason I, Reynolds AP, Catt SD, Peters TJ, Menzies IS. Lactulose, $51 \mathrm{Cr}$-labelled ethylenediaminetetraacetate, L-rhamnose and polyethylene glycol 400 as probe maters for assessment in vivo of human intestinal permemarkers for assessment in vivo of

3 Pearson ADJ, Eastham EJ, Laker MF, Craft AW, Nelson R Intestinal permeability in children with Crohn's disease and coeliac disease. $\mathrm{Br}$ Med $f$ 1982; 285: 20-1.

4 Bjarnason I, O'Morain C, Levi AJ, Peters TJ. Absorption of 51 Chromium-labelled ethylenediamine-tetra-acetate in inflammatory bowel disease. Gastroenterology 1983; 85: $318-22$
5 Menzies IS, Laker MF, Pounder R, et al. Abnormal intestinal permeability to sugars in villous atrophy. Lancet 1979; ii: permeabilit

6 Bjarnason I, Peters TJ, Veall N. A persistent defect in intestinal permeability in coeliac disease demonstrated by a 51Cr-labelled EDTA absorption test. Lancet 1983; i: 323-5.

7 Bjarnason I, Williams P, So A, et al. Intestinal permeability and inflammation in rheumatoid arthrits: effects of non-steroidal anti-inflammatory drugs Lancet 1984; ii: 1171-3.

8 Bjarnason I, Zanelli G, Smith T, et al. Nonsteroidal antiinflammatory drug-induced intestinal inflammation in inflammatory drug-induced intestinal

9 Rampton DS, McNeill NI, Sarner M. Analgesic ingestion and other factors preceding relapse in ulcerative colitis. $G u$ 1983; $24: 187-9$.

10 Kaufmann HJ, Taubin HL. Nonsteroidal anti-inflammatory drugs activate quiescent inflammatory bowel disease. Ann Intern Med 1987; 107: 513-6.

11 Langman MJS, Morgan L, Worrall A. Use of antiinflammatory drugs by patients admitted with small or large bowel perforations and haemorrhage. Br Med 7 1985; 290 $347-9$.

12 Bjarnason I, Zanelli G, Prouse P, Williams P, Gumpel MJ, Levi AJ. Effect of non-steroidal anti-inflammatory drugs on the human small intestine. Drugs 1986; 32: 35-41.

13 Elia $M$, Behrens $R$, Northrop $C$, Wraight P, Neale G. Evaluation of mannitol, lactulose and $5 \mathrm{iCr}$-labelled ethylenediaminetetra-acetate as markers of intestinal permeability in man. Clin Sci 1987; 73: 197-204.

14 Vogelsang H, Ferenci P, Frotz S, Meryn S, Gangl A. Acidic colonic microclimate - possible reason for false negative hydrogen breath tests. Gut 1988; 29: 21-6.

15 Menzies IS, Mount JN, Wheeler MJ. Quantitative estimation of clinically important monosaccharides in plasma by rapid thin-layer chromatography. Ann Clin Biochem 1978; 15 65-76.

16 Noone C, Menzies IS, Banatvala JE, Scopes JW. Intestinal permeability and lactose hydrolysis in human rotaviral
gastroenteritis assessed simultaneously by non-invasive gastroenteritis assessed simultaneously by non-invasive differential

17 Black DA. Intestinal permeability in the elderly. Dig Dis $S_{c i}$ 1988; 33: 382

18 Borriello SP. Bacteria and gastrointestinal secretion and motility. Scand $\mathcal{F}$ Gastroenterol 1984; 19 (suppl 93): 115-21.

19 Maxton DG. Studies on small intestinal absorption and permeability. MD thesis. London: University of London, 1986.

20 Nissim JA. Reduction of intestinal absorption by a synthetic chemical Nature $1960 ; 185: 222-4$.

21 Holgate AM, Read NW. Relationship between small bowel transit time and absorption of a solid meal. Influence of transit time and absorption of a solid meal. Influence of metoclopramide, magn

22 Robert A, Asano T. Resistance of germ free rats to indomethacin-induced intestinal lesions. Prostaglandins 1977; 14: 333-41.

23 Reynold JEF, ed. Martindale. The extra pharmacopoeia. 28th edition. London: The Pharmaceutical Press, 1982. 